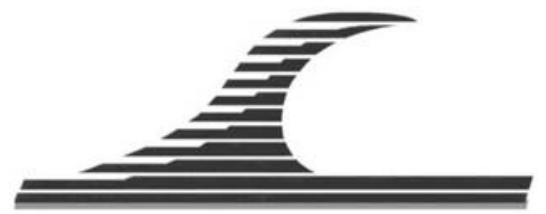

Revue Paralia, Volume 8 (2015) pp n01.1-n01.16

Mots-clés : Erosion et accrétion, Bilan sédimentaire, Profil de plage, Port-Bouët, Jacqueville, Côte d'Ivoire.

(C) Editions Paralia CFL

\title{
Effets de l'ouverture du Canal de Vridi sur les stocks sédimentaires littoraux entre Abidjan et Jacqueville (Côte d'Ivoire)
}

\author{
Gnosseith N'DOUFOU ${ }^{1}$, Jacques ABE ${ }^{1}$, Siaka BAMBA ${ }^{1}$, \\ Célestin HAUHOUOT ${ }^{2}$, Kouamé AKA ${ }^{3}$
}

1. Département Environnement, Laboratoire de Physique et de Géologie Marine, Centre de Recherches Océanologiques d'Abidjan, BP V18, Abidjan, Côte d'Ivoire. huberson7@hotmail.fr ; jabe1@hotmail.com ; Bambasb@hotmail.com

2. Institut de Géographie Tropicale, Université de Cocody, 22 BP 744, Abidjan, Côte d'Ivoire.

C_hauhouot@yahoo.fr

3. Laboratoire de Géologie marine et de sédimentologie, UFR-Sciences de la Terre et des Ressources Minières, Université de Cocody, 22 BP 582 Abidjan, Côte d’Ivoire. akaraphael@yahoo.fr

\section{Résumé :}

Ce travail est une contribution à la connaissance des modifications morpho sédimentaires du littoral compris entre Abidjan et Jacqueville. La méthode utilisée à cette fin s'appuie sur le traitement, l'analyse de profils topographiques et d'échantillons de sable collectés sur le terrain lors de campagnes successives effectuées entre 1998 et 2008. Cette approche a débouché sur la mise en évidence d'un déficit sédimentaire de $2500 \mathrm{~m}^{3}$ à Port-Bouët et d'un bilan sédimentaire positif de $+2300 \mathrm{~m}^{3}$ à Jacqueville. Cet engraissement occasionne une progradation de $13,5 \mathrm{~m}$ du trait de côte. L'étude sédimentologique met en évidence des stocks sédimentaires différents sur les deux sites. Un stock constitué principalement de matériels polis et grossiers à Jacqueville qui dénote un long transport par l'eau, et un stock constitué essentiellement de matériels subanguleux à anguleux et très grossiers à Port-Bouët témoignant d'une source pourvoyeuse proche. Cette étude met en évidence les effets des ouvrages de protection du canal portuaire sur la répartition des faciès entre Jacqueville et Port-Bouët.

Soumis le 11 janvier 2010, accepté le 16 janvier 2015, en ligne le 2 février 2015.

La seule version examinée est celle écrite en français. La ou les autres versions n'étant pas examinées par le comité de rédaction de la revue, sont donc publiées sous l'entière responsabilité du ou des auteurs.

A TRANSLATED VERSION IN ENGLISH IS AVAILABLE ONLINE

Pour citer cet article :

N’DOUFOU G., ABE J., BAMBA S., HAUHOUOT C., AKA K. (2015). Effets de l'ouverture du Canal de Vridi sur les stocks sédimentaires littoraux entre Abidjan et Jacqueville (Côte d'Ivoire). Revue Paralia, Vol. 8, pp n01.1-n01.16.

DOI: http://dx.doi.org/10.5150/revue-paralia.2015.n01 


\section{Introduction}

L'érosion côtière est un phénomène majeur dans le monde. Elle affecte la moitié des rivages maritimes de la terre. De plus en plus les activités humaines amplifient ce phénomène. En Côte d'Ivoire, le canal de Vridi en est une illustration. En effet, avant la percée du canal de Vridi, le dépôt de sable au niveau de la baie de Port-Bouët du fait de la dérive littorale ouest-est, entrainait une progradation du trait de côte de l'ordre de $1 \mathrm{~m} \mathrm{an}^{-1}$ (PELNARD, 1973). Cette tendance est inversée après la construction des musoirs ouest et est, respectivement en 1943 et 1950, puis de la digue d'arrêt des sables en 1973 (TASTET et al., 1985). Ce secteur en proie au recul renferme les principales infrastructures économiques du pays dont l'aéroport et surtout le Port Autonome d'Abidjan qui constitue le $1^{\mathrm{er}}$ poumon économique du pays.

Cette étude fait suite à des travaux précédents réalisés sur ce segment de côte (TASTET et al., 1985 ; TASTET, 1987 ; KOFFI et al., 1987 ; YACE, 1987 ; HAUHOUOT, 2000 ; $\mathrm{ABE}, 2005)$. La dernière étude avait montré que la dynamique dans la zone de l'épave du Dora subissait l'influence des structures de protection du Port à l'entrée du canal de Vridi avec une progradation de l'ordre de 50 m entre janvier 1986 et septembre 2004. Mais cette étude comme les travaux précédents n’apporte pas suffisamment d'information sur la dynamique de la plage de Jacqueville. Dès lors, pour pallier ce déficit d'information et obtenir une idée assez précise quant à l'évolution de cette plage, nous avons initié cette étude. En outre, le périmètre littoral de Port-Bouët est un milieu où les changements sont parfois rapides. Ceci constitue une menace sérieuse pour les riverains et les autorités. En effet, le recul du rivage inhérent aux installations portuaires a pour corollaire la destruction des habitats et la réduction de l'espace constructible. Il devient par conséquent nécessaire d'actualiser les connaissances sur la dynamique sédimentaire de ce secteur à fort enjeu socio-économique. Cette étude complémentaire permettra de combiner nos résultats actuels avec ceux des travaux antérieurs pour ressortir les tendances évolutives utiles à la gestion du littoral.

\section{Présentation de la zone d'étude}

\subsection{Cadre géologique et morphologique}

La zone d'étude (figure 1) appartient au bassin sédimentaire ivoirien qui représente $3 / 5$ de la façade atlantique du pays (TASTET, 1972). Il couvre une superficie de $30000 \mathrm{~km}^{2}$ dont la plus grande partie est immergée. La partie émergée forme un croissant entre Sassandra et le Ghana (DELOR et al., 1992). Elle est traversée d'ouest en est par une faille importante (TASTET, 1979). Au nord de la faille, les formations superficielles sont constituées d'argiles, de sables argileux et de grès plus ou moins ferrugineux du continental terminal (DELOR et al., 1992). 


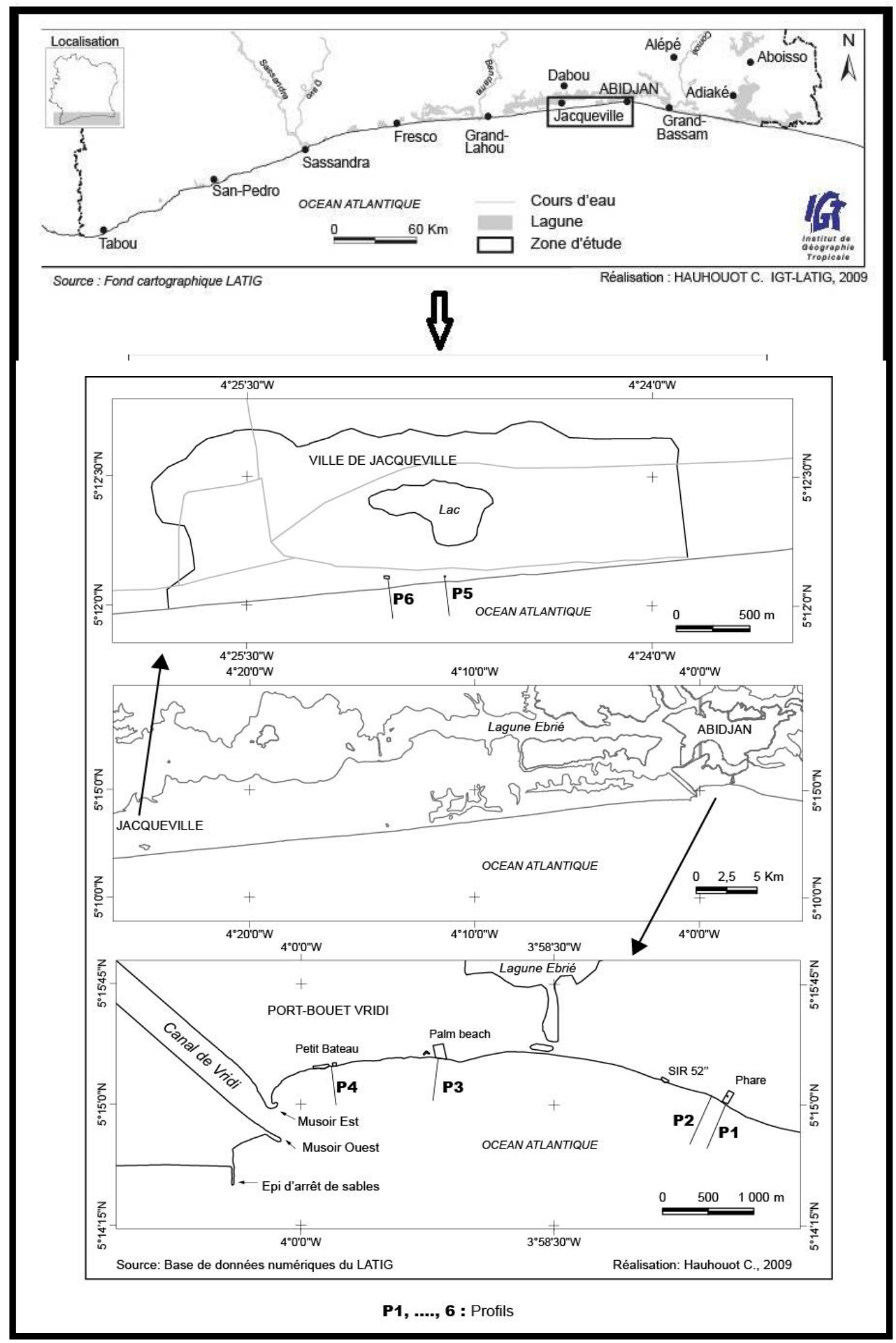

Figure 1. Localisation de la zone d'étude. 
Au sud, les formations superficielles sont des dépôts quaternaires de sables et d'argiles continentaux, de sables blancs et d'argiles fluvio-lagunaires et de sables marins (DELOR et al., 1992). D’un point de vue géomorphologique, les formations du continental terminal constituent des "Haut plateaux" d'altitude variant entre $50 \mathrm{~m}$ et $110 \mathrm{~m}$ tandis que les dépôts quaternaires forment une plaine littorale basse au sud des lagunes. De l'extrémité ouest de la lagune de Grand-Lahou jusqu'au Ghana, le trait de côte est formé par une plage sableuse adossée à des cordons littoraux d'épaisseur variable. A l'ouest d'Abidjan, les cordons forment une bande sableuse étroite de quelques centaines de mètres. A partir d'Abidjan, ils s'étendent vers l'intérieur sur plus de $4 \mathrm{~km}$ de large. Le rivage entre Sassandra et Abidjan est orienté dans le $81^{\circ}$ (ABE \& KABA, 1997). Au niveau d'Abidjan, il change de direction et s'oriente dans le $100^{\circ}$ jusqu'au Ghana (ABE, 2005). Ce changement d'orientation divise notre zone d'étude en deux secteurs dynamiques différents si l'on considère l'angle d'attaque de la houle. Ces deux secteurs renferment des plages réflectives (HAUHOUOT, 2000 ; ABE, 2005). Cet état de plage n'est pas favorable à la construction des barres sableuses sous-marines ou intertidales (DESMAZES, 2000 ; 2001). A Port-Bouët, la plage est globalement uniforme. En revanche, à Jacqueville des bancs rocheux continus ont été identifiés au niveau de la plage sous-marine (MARTIN, 1973; AKA, 1991 ; MONDE, 1997) (figure 2).

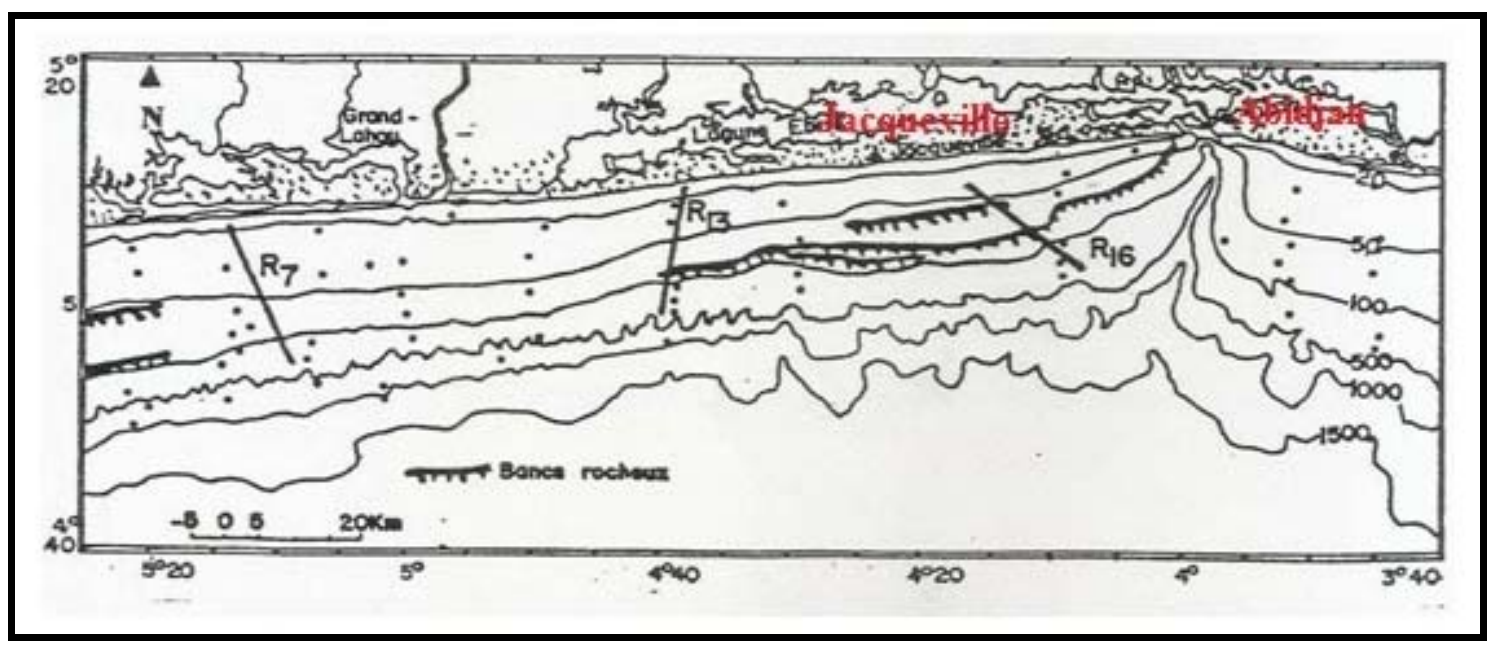

Figure 2. Profils bathymétriques du plateau continental de Côte d'Ivoire (AKA, 1991).

\subsection{Les agents dynamiques}

Dans la région, le rivage est battu fréquemment par des houles d'amplitude comprise entre 0,80 et 2,00 m et de période 10 à 12 secondes. Exceptionnellement, des houles de plus grande amplitude pouvant atteindre des périodes de $20 \mathrm{~s}$ déferlent sur la côte (VARLET, 1958). Elles sont très destructrices. Les houles qui déferlent habituellement dans la région, malgré leurs caractéristiques moyennes, ont des effets morphologiques 
significatifs sur le trait de côte dans le contexte dynamique actuel. Elles abordent le rivage avec une direction préférentielle sud-ouest à sud. Les houles orientées sud-sudouest aborde la section de côte Grand-Lahou-Abidjan avec un angle de $60^{\circ}$ (HINSCHBERGER, 1977) de sorte qu'il se produit une forte dérive littorale estimée à $800000 \mathrm{~m}^{3} \mathrm{an}^{-1}$ de sable (MARTIN, 1973). C'est donc une section de côte où domine le transport sédimentaire. C'est aussi une côte où l'érosion est sensible car, si l'on considère que cette section reçoit environ $200000 \mathrm{~m}^{3} \mathrm{an}^{-1}$ de sables provenant de la côte ouest (Tabou-Sassandra), on comprend que la différence provient des stocks sédimentaires de la région (MARTIN, 1973). La régularisation du rivage, la présence "permanente" de micro falaise (2 à $3 \mathrm{~m}$ ) et la destruction des constructions sur l'arrièreplage qu'on observe dans la région sont d'autres effets de l'érosion côtière.

A l'est d'Abidjan, les houles sud-sud-ouest abordent la côte avec un angle de $85^{\circ}$ (HINSCHBERGER, 1977). Elles sont presque perpendiculaires à la côte ce qui amoindrit sensiblement l'efficacité de la dérive littorale. Cette zone est favorable aux dépôts ; l'émergence de dérives en sens contraire engendrées par les houles du sud contribue aussi à l'accumulation. Toutefois, devant Abidjan, l'entaille du Trou-sansfond (nom donné au canyon sous-marin qui entaille le plateau continental devant Abidjan) et l'aménagement du canal de Vridi rendent beaucoup plus complexe la dynamique sédimentaire. Les caractéristiques générales des houles devant Abidjan sont consignées dans le tableau 1. Les autres agents marins ont peu d'incidence sur la dynamique côtière. Le courant superficiel de Guinée et son contre-courant n’agissent pas directement sur la morphologie du trait de côte. Ils redistribuent sur le plateau continental les particules fines expulsées en mer par les fleuves. Le courant de Guinée est efficace entre zéro et trente mètres. Il étale les particules vers l'est. Au-delà de trente mètres, le contre-courant piège les particules, il les déplace dans le sens contraire et il les abandonne à l'ouest des embouchures (MARTIN, 1973). La marée en Côte d'Ivoire est de type semi-diurne avec un marnage d'un mètre (VARLET, 1958). Il est trop faible pour influencer de façon significative la morphologie côtière. Tout au plus les courants associés à la marée remodèlent la pointe des flèches qui se forment dans les passes. Le marnage relevé au niveau du Port d'Abidjan varie entre 0,40 m, en mortes-eaux et 1,30 m en vives-eaux (VARLET, 1958).

Tableau 1. Caractéristiques de la houle devant Abidjan (TASTET et al., 1985).

\begin{tabular}{llllll}
\hline Houle & $\begin{array}{l}\text { Amplitude } \\
(\mathrm{m})\end{array}$ & $\begin{array}{l}\text { Période } \\
(\mathrm{s})\end{array}$ & $\begin{array}{l}\text { Direction } \\
\text { au large }\end{array}$ & $\begin{array}{l}\text { Fréquence } \\
\text { annuelle }\end{array}$ & $\begin{array}{l}\text { Epoque } \\
\text { de dominance }\end{array}$ \\
\hline Faible & $0,8-1$ & $7-11$ & $S-S W$ & $30 \%$ & Nov. - Déc. Jan. \\
Moyenne & $1-2$ & 10 & $S-S W$ & $50 \%$ & Toute l'année \\
Forte & $1,8-2$ & $10-20$ & $S-S W$ & $20 \%$ & Mai - Juin \\
\hline
\end{tabular}




\section{Matériel et méthode de travail}

Le travail de terrain réalisé en 2006, 2007 et 2008, a consisté à lever les profils perpendiculaires au rivage à l'aide d'un niveau, d'une mire de $4 \mathrm{~m}$ et d'un trépied.

Les levés ont été effectués de manière à pouvoir les comparer avec les données antérieures obtenues à partir de profils topographiques calés sur des repères de référence. Ces données datant de 1998 jusqu’ici inexploitées ont été fournies par le Centre de Recherches Océanologiques d'Abidjan. Pour ce qui concerne le secteur de Port-Bouët, le profil de mars 1990 a été associé pour enrichir la connaissance. Le manque de données correspondant à cette période pour le secteur de Jacqueville n’enlève rien à l'interprétation des autres profils. Les levés ont été effectués à marée basse pour explorer une largeur maximale de la plage. Dans cette étude, l'ados de plage a été pris comme ligne de référence représentative du trait de côte. Dans le cas des côtes sableuses, l'ados de plage s'avère être le plus fiable (ROBIN, 2002). Il est matérialisé par une rupture de pente facilement identifiable (figure 3). Les bilans sédimentaires sont établis à partir de la détermination des superficies érodées et/ou engraissées par la méthode des trapèzes (BRABANT, 2003). Des prélèvements superficiels d'échantillons de sable ont été effectués lors de chaque opération de levés de profils sur la haute plage et le bas estran pour des études granulométriques et morphoscopiques afin de vérifier s’il existe des différences sédimentologiques significatives du matériel des plages. L'analyse granulométrique des sables a été effectuée après tamisage pendant $15 \mathrm{mn}$ à travers une colonne de 13 tamis répondant aux normes AFNOR. Les résultats ont été interprétés selon les démarches sédimentologiques classiques (FOLK \& WARD, 1957 ; FRIEDMAN, 1967). L'analyse morphoscopique a consisté à trier les fractions granulométriques comprises entre 250 à $400 \mu \mathrm{m}$, taille de référence proposée par CAILLEUX (1943) et à les classer après observation à la loupe binoculaire en tenant compte de deux critères : la forme des grains et leur état de surface. L'estimation de la sphéricité et de l'émoussé des grains de quartz a été faite selon l'échelle de Power (1953, in ROCHELEAU, 1997). Pour mieux visualiser la forme et l'aspect des grains de quartz nous avons procédé à des analyses au microscope électronique à balayage (MEB). Le MEB de type FEG SUPRA 40 VP Zeiss est équipé d'un système de microanalyse $X$ et de spectrométrie par dispersion d'énergie (EDS). Le traitement des échantillons au MEB a consisté à balayer la surface des grains de quartz par un faisceau d'électron. En réponse, ces grains ont émis certaines particules. Ces particules ont ensuite été analysées par le détecteur EDS. Ceci a permis de reconstruire une image en trois dimensions de la surface des grains de quartz. Ces analyses au MEB ont été nécessaires pour obtenir des informations assez précises pour ce qui a trait à l'origine du matériel sableux. 


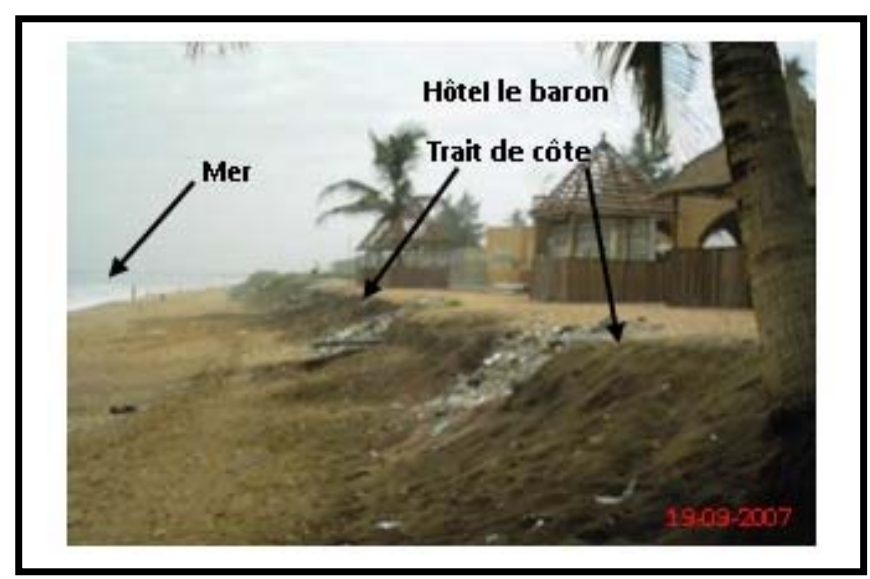

Figure 3. Menace d'érosion à la hauteur de l'hôtel le Baron à Port-Bouët.

\section{Résultats et interprétation}

\subsection{Evolution morphologique des plages}

\subsubsection{Evolution de la plage de Jacqueville}

A Jacqueville, les profils ont une allure convexe et témoignent d'une zone favorable à l'accumulation (figure 4). Ce secteur gagne du matériel sableux estimé à $+2300 \mathrm{~m}^{3}$. Ce qui se traduit par une avancée du trait de côte qu'on estime à 13,5 m. La dynamique dans ce secteur situé en aval, dérive de la plage de Grand-Lahou en érosion, est difficile à expliquer ; ces deux plages étant situées sur une portion de côte sableuse parfaitement rectiligne, orientée dans le $81^{\circ}$ (TASTET, 1972). En effet, le périmètre littoral de Grand-Lahou subit un recul moyen d'environ $1 \mathrm{~m} \mathrm{an}^{-1}$ (N'DOUFOU, 2005). Les pertes enregistrées dans le secteur de l'embouchure ont été estimées à 10,7 ha entre 1993 et 2006 (HAUHOUOT, 2008). On peut donc émettre l'hypothèse d'une alimentation de la plage de Jacqueville par les apports des secteurs voisins en érosion, notamment la plage de Grand-Lahou. A l'instar des barres sableuses qui jouent un rôle essentiel dans la dynamique des plages, tout particulièrement vis-à-vis de l'érosion et des modifications de la côte lors des tempêtes (LIPPMANN \& HOLMAN, 1990), les bancs rocheux continus situés au niveau de la plage sous-marine à Jacqueville (figure 2) devraient atténuer l'effet des vagues et confirmer une telle hypothèse. Ce phénomène a déjà été décrit dans d'autres régions littorales, entre autres sur les côtes ghanéennes (AKOBE, 2010). La plage de Jacqueville ne devrait logiquement pas être influencée par des ouvrages situés à une quarantaine de kilomètres. En outre, des témoignages recueillis sur le terrain révèlent que cette dynamique est antérieure aux premiers aménagements au niveau du canal. 


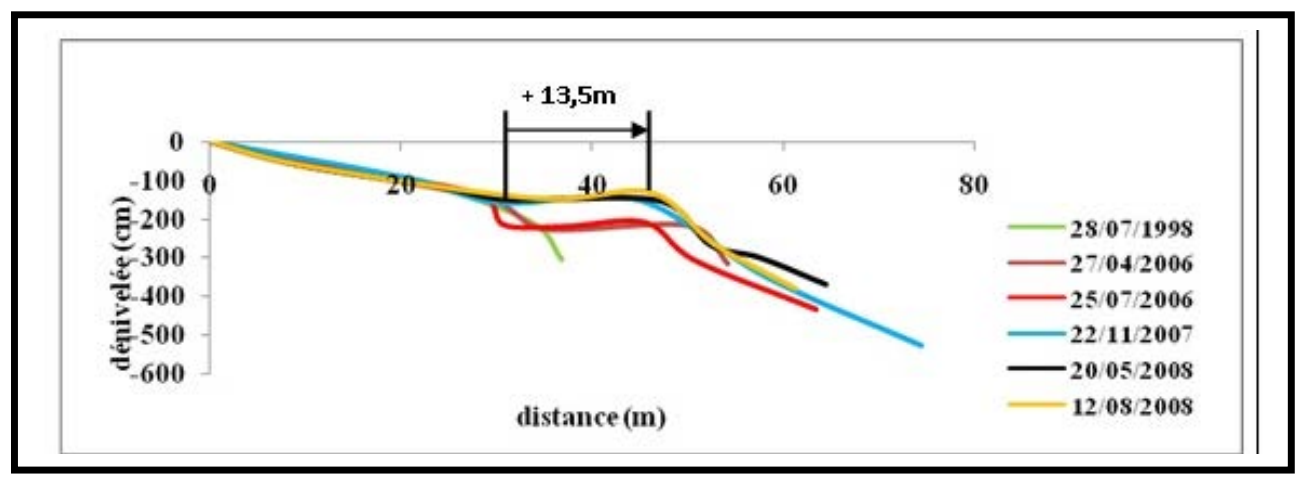

Figure 4. Evolution de la plage de Jacqueville.

\subsubsection{Evolution de la plage de Port-Bouët.}

A Port-Bouët, les profils ont pour la plupart une allure concave. La superposition des profils montre deux grandes périodes quant à l'évolution du trait de côte (figure 5). De mars 1990 à juillet 1998 on enregistre une forte érosion de la plage qui imprime un recul de 7,6 m du trait de côte. Dans ce secteur, les installations portuaires réduisent les stocks sédimentaires mobilisables sur le rivage et accélèrent l'érosion. La période suivante (juillet 1998 à septembre 2008) voit l'érosion se poursuivre, notamment au bas de plage. Le bilan sédimentaire est négatif et est estimé à $-2500 \mathrm{~m}^{3}$. Le trait de côte reste cependant inchangé, la haute plage étant quasiment stable. On peut donc considérer qu'une diminution du stock sableux n’entraine pas nécessairement un recul du rivage. Ce qui peut paraître surprenant. En effet, le trait de côte a subi un recul moyen d'environ $1 \mathrm{~m} \mathrm{an}^{-1}$ entre 1990 et 1998 avant de se stabiliser au niveau de la route du fait de la résistance des roches (bitume, gravier, ...) ayant servi à la réalisation de cette infrastructure (figures 5 et 6). La stabilité du trait de côte dans ce secteur réputé instable (TASTET et al., 1985 ; HAUHOUOT, 2000 ; ABE, 2005) pourrait s'expliquer ainsi. Entre juillet 1998 et septembre 2008, les mouvements sédimentaires les plus importants se sont déroulés sur le bas de plage. Ceci semble normal car cette partie de plage est soumise aux effets combinés de la marée et des vagues (DESMAZES, 2005). Ce sont les facteurs hydrodynamiques les plus importants qui influencent et déterminent le comportement dynamique des plages (IBE \& QUELENNEC, 1989). En outre, le bas de plage est la zone où l'énergie des houles est la plus forte (ZEIGLER \& TUTTLE, 1961). Entre septembre 2007 et novembre 2007, la plage se reconstitue progressivement. Cette dynamique correspond aux mois où la houle est la moins agitée et illustre un mouvement sédimentaire des sables vers la côte. La reconstitution est interrompue entre mai et juillet 2008. Durant cette période, la mer est très agitée (TASTET et al., 1985). Les phénomènes naturels contribuent considérablement au changement morphosédimentaire de la plage. Ce secteur a enregistré durant la période d'étude, de violentes tempêtes notamment en août 1993, en avril 1997, en août 2007 (figure 7). Si l'on se réfère à la fréquence des tempêtes, une déduction s'impose: celle de l'impact 
prépondérant possible sur ce segment de côte, d'où la nécessité de relater ces informations car elles s'avèrent très utiles à la compréhension de l'évolution de cette plage. En 1984, le phénomène qui n’a duré qu'une nuit a fait reculer le trait de côte de $20 \mathrm{~m}$ au Phare de Port-Bouët (ABE \& N'GUESSAN, 1995). La plage de Port-Bouët a un fonctionnement complexe car il est difficile de faire la part entre les dynamiques marines (en particulier les tempêtes) et l'action des équipements côtiers (digue et épis) sur le long terme.

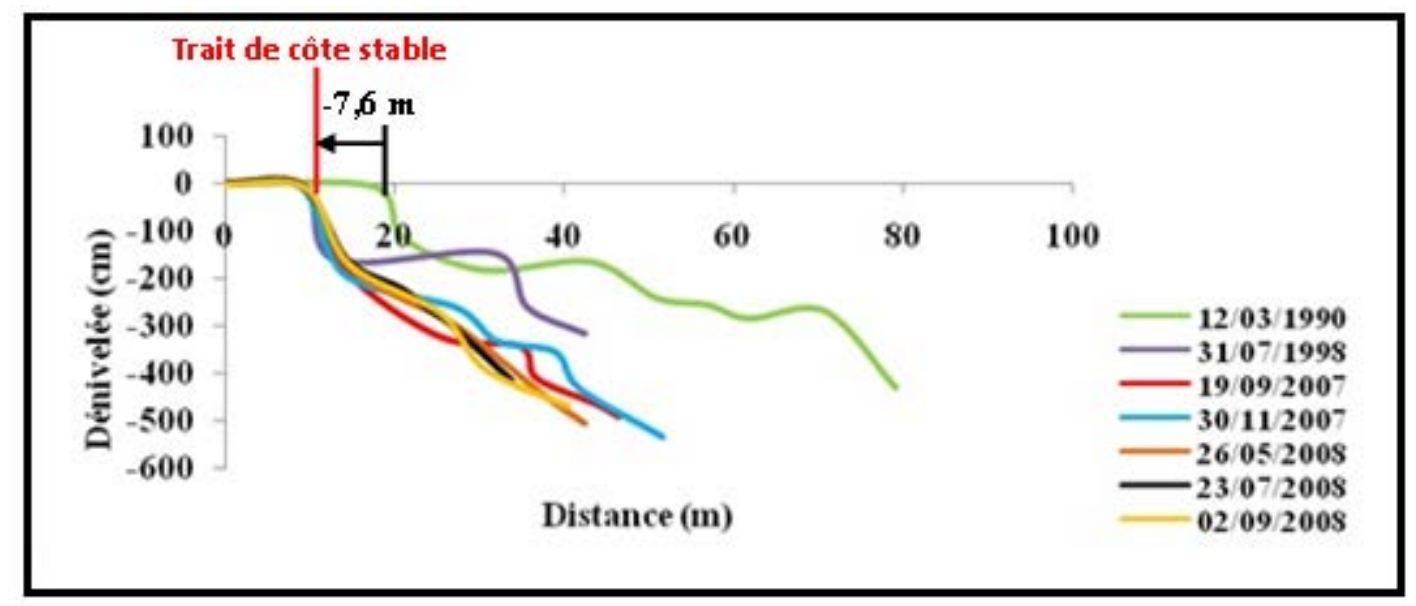

Figure 5. Evolution de la plage de Port-Bouët.

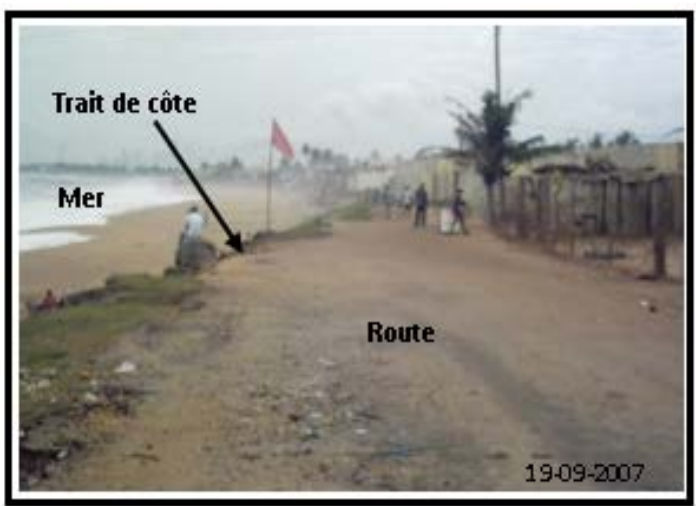

Figure 6. Route interrompue par l'érosion au Phare de Port-Bouët.

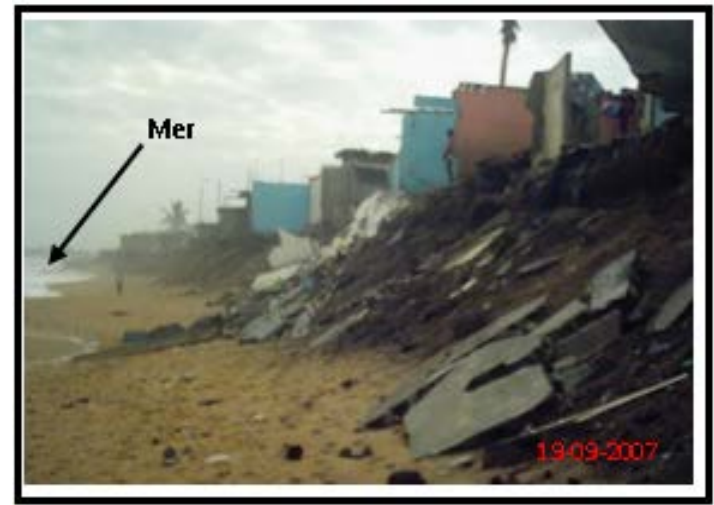

Figure 7. Dégâts causés par la tempête d'août 2007 à l'ouest du Phare.

\subsection{Etude sédimentologique des plages}

\subsubsection{Granulométrie à l'ouest du canal de Vridi}

A Jacqueville, la distribution granulométrique des échantillons de sable le long des profils montre que les sédiments sont modérément classés $0,53<\sigma<0,62$ avec une abondance de sables grossiers $592<\mathrm{Mz}(\mu \mathrm{m})<626$. Les sables de plage sont symétriques $0,00<\mathrm{Sk}<0,01$ (voir tableau 2). 


\subsubsection{Granulométrie à l'est du canal de Vridi}

A proximité du canal (Palm Beach et Petit Bateau, voir tableau 2), les sables sont très grossiers $(1078<\mathrm{Mz}(\mu \mathrm{m})<1419)$, bien classés $(0,42<\sigma<0,47)$ et symétriques $(-0,03<\mathrm{Sk}<0,04)$.

Au Phare (voir tableau 2), les sables sont grossiers $(764<\mathrm{Mz}(\mu \mathrm{m})<821)$. Les sédiments du bas estran sont symétriques et bien classés. Par contre ceux du haut estran sont modérément classés avec une forte asymétrie vers les éléments grossiers. Le classement des particules diminue sensiblement vers la haute plage, signalant qu'un tri ou un vannage s'est effectué au fur et à mesure du dépôt. Les sédiments très grossiers rencontrés à Port-Bouët témoignent d'un environnement à forte énergie. Dans ce secteur, la proximité du canyon amplifie l’énergie des vagues (TASTET et al., 1985).

Tableau 2. Paramètres granulométriques des sables à Port-Bouët et à Jacqueville.

\begin{tabular}{|c|c|c|c|c|}
\hline & & $\begin{array}{l}\text { Moyenne } \\
(\mu \mathrm{m})\end{array}$ & $\begin{array}{l}\text { Ecart-type } \\
(\sigma)\end{array}$ & $\begin{array}{l}\text { Skewness } \\
\text { (Sk) }\end{array}$ \\
\hline Canal est & P2 HE & 821 & 0,58 & $-0,38$ \\
\hline \multirow[t]{11}{*}{ Port-Bouët } & Phare & & & \\
\hline & $P 2 B E$ & 764 & 0,48 & 0,04 \\
\hline & Phare & & & \\
\hline & P3 HE & 1078 & 0,48 & 0,04 \\
\hline & Palm Beach & & & \\
\hline & P3 BE & 1419 & 0,42 & $-0,03$ \\
\hline & Palm Beach & & & \\
\hline & P4 HE & 1362 & 0,43 & $-0,02$ \\
\hline & Petit Bateau & & & \\
\hline & P4 BE & 1183 & 0,47 & 0,05 \\
\hline & Petit Bateau & & & \\
\hline Canal & P5 HE & 592 & 0,62 & 0,01 \\
\hline ouest & Monument & & & \\
\hline \multirow[t]{2}{*}{ Jacqueville } & P5 BE & 626 & 0,53 & 0,00 \\
\hline & Monument & & & \\
\hline
\end{tabular}

L'étude granulométrique montre que les sédiments sont plus grossiers à l'est du canal, donc dans le sens du transport sédimentaire (figure 8) à l'inverse de ce que propose le modèle de MC LAREN (1981). Cette répartition des faciès observés de part et d'autre du canal de Vridi serait la résultante de deux phénomènes : un changement dans la dynamique du milieu à Port-Bouët, dynamique influencée par la présence du trou-sansfond ; un blocage des sédiments provenant de l'ouest au niveau de la digue d'arrêt des 
sables et une nouvelle source d'alimentation à partir du périmètre littoral de Port-Bouët et ce d'autant que selon le modèle de Mc LAREN (1981), la taille des sédiments s'affine dans le sens du transport.

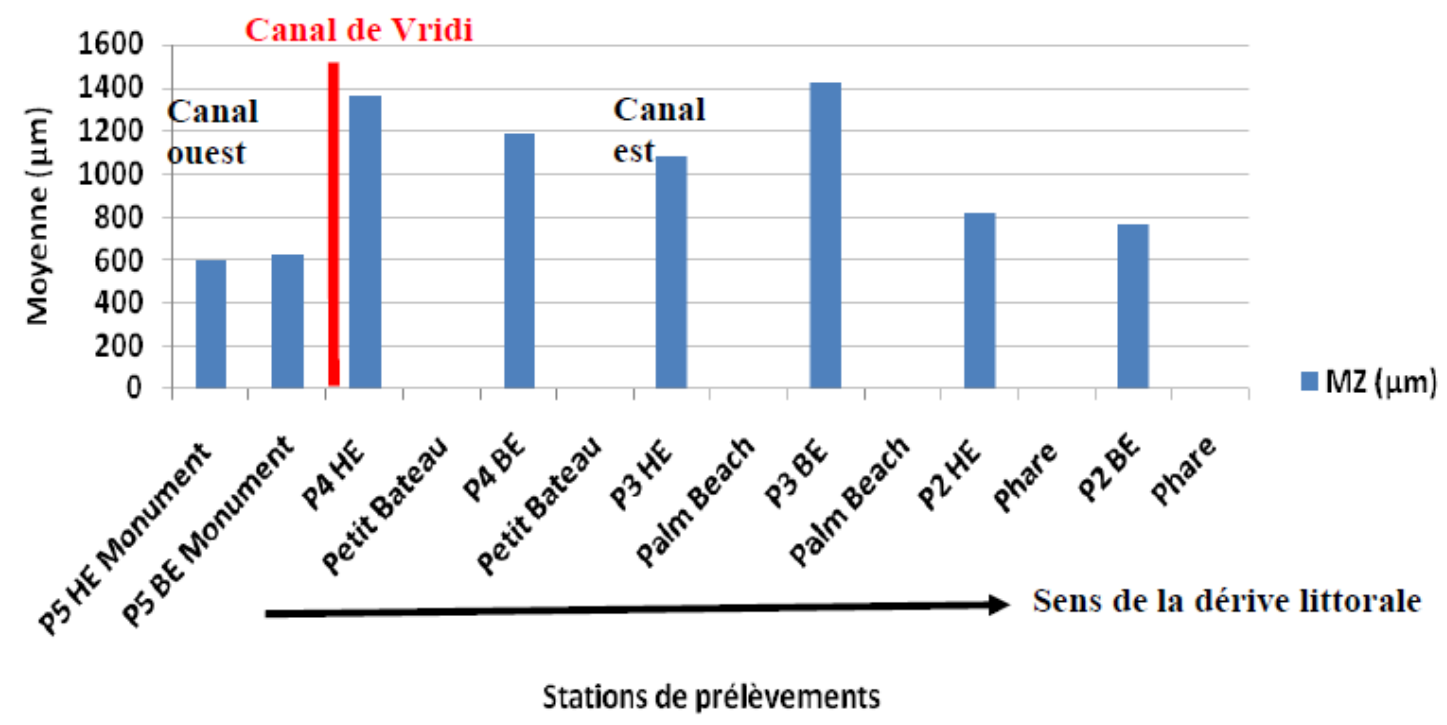

Figure 8. Variation de la moyenne sur l'estran à l'est et à l'ouest du Canal de Vridi.

\subsubsection{Morphoscopie et exoscopie des quartz à l'ouest du canal de Vridi}

L'examen morphoscopique et exoscopique montre que les grains de quartz sont généralement subarrondis à arrondis et luisants (figures 9a et 9b). Leur proportion oscille entre 70 et $80 \%$. On distingue des figures de frottement polies par des reprises aquatiques (figure 9d). Ce polissage tend à effacer les traces de chocs. Cela peut s'expliquer par un séjour prolongé dans la zone d'action des houles. Les ronds mats (figure 9c) ont été observés conjointement avec les émoussés luisants. Ils sont peu représentés (10 à 15\%) et témoignent d'un transport sur une longue distance (SAAIDI, 1991). L'aspect mat est produit par suite de nombreux chocs des grains entre eux (VATAN, 1967). Certains auteurs attribuent cet aspect à une cimentation de calcite (LINDHOLM, 1987). Les non usés se présentent en très faible proportion (<3\%).

\subsubsection{Morphoscopie et exoscopie des quartz à l'est du canal de Vridi}

Les grains de quartz sont pour la plupart subanguleux à anguleux (65 à 83\%) (figures $10 \mathrm{a}$ et $10 \mathrm{~d})$ accompagnés accessoirement d'éléments subémoussés et luisants ( $<5 \%)$ (figure 10b). Les quartz dont le contour est anguleux caractérisent un transport sur une courte distance (SAAIDI, 1991). Les grains très arrondis sont observés conjointement avec les non (ou peu) usés (figure 10c). Leur proportion oscille entre 1 et $2 \%$. Ces grains très arrondis qui caractérisent un transport sur une longue distance ont dû échapper à la digue d'arrêt des sables compte tenu de leur très faible proportion. 


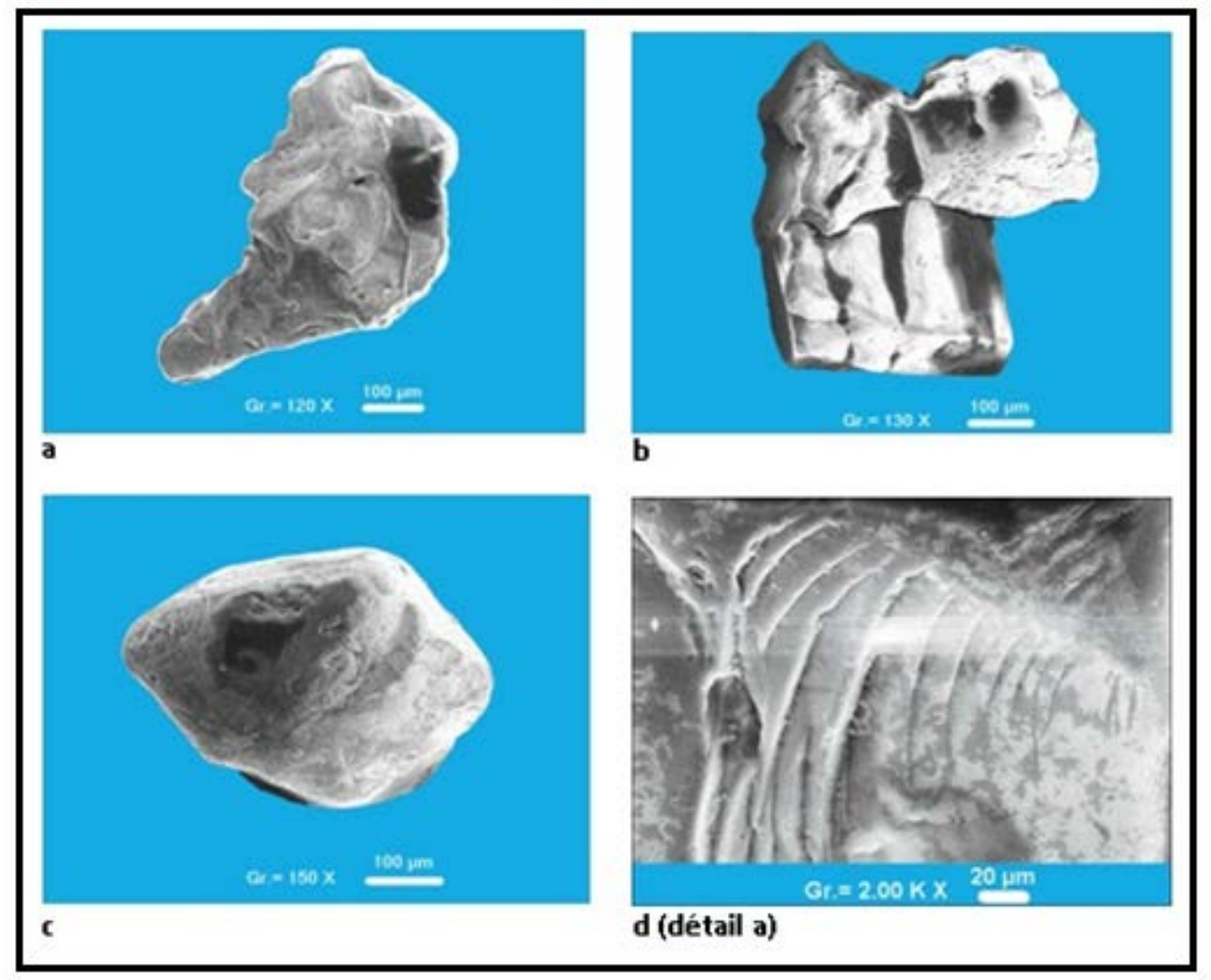

Figure 9. Grains de quartz à l'ouest du canal de Vridi (Jacqueville).

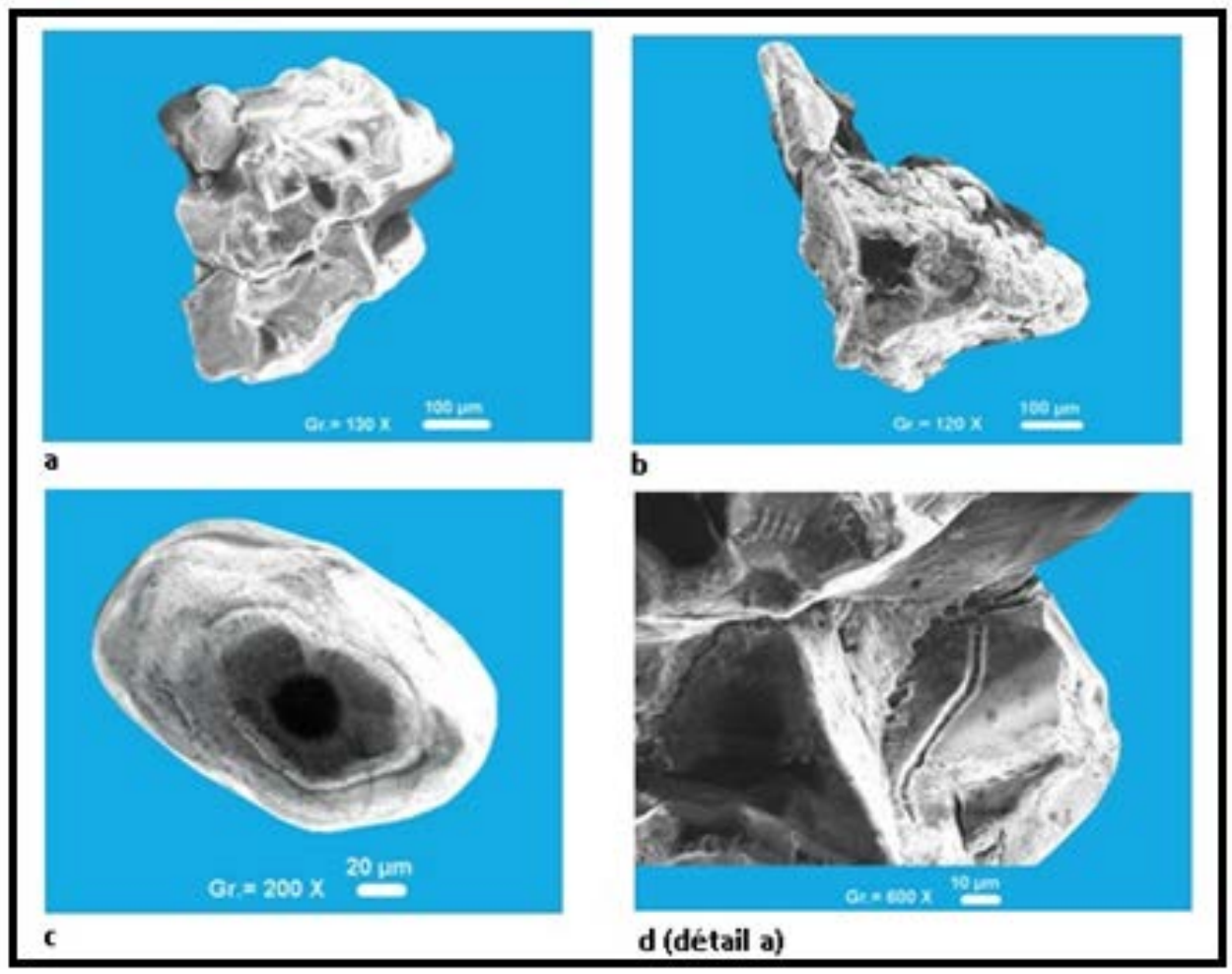

Figure 10. Grains de quartz à l'est du canal de Vridi (Port-Bouët). 


\section{Discussion-conclusion}

L'étude comparée des deux sites permet de mettre en évidence deux secteurs morphosédimentaires distincts. L'un à l'est du canal, peu alimenté par le déplacement latéral du matériel sableux, en érosion $\left(-2500 \mathrm{~m}^{3}\right)$. Ce secteur subit la conséquence de l'interruption du transit sédimentaire ouest-est au niveau de la digue d'arrêt des sables avec un recul moyen de $1 \mathrm{~m} \mathrm{an}^{-1}$. Les travaux de TASTET et al. (1985) ont montré une érosion du littoral à l'est du canal de Vridi et un engraissement à l'ouest depuis la construction de la digue d'arrêt des sables en 1973. Ces observations ont été confirmées par YACE (1987), HAUHOUOT (2000) et ABE (2005). Les résultats de la présente étude sont en harmonie avec les travaux précités. Cependant, dans cette étude l'évolution de la plage est marquée par une stabilité de la ligne de rivage entre 1998 et 2008 alors que les travaux antérieurs décrivent plutôt un recul continu du rivage. En effet, le schéma classique de fonctionnement propose un recul du trait de côte pendant les tempêtes, recul consécutif au déplacement des sables vers le large (STIVE \& DEVRIEND, 1995). Dans notre étude, la position du trait de côte est relativement stable alors que la tendance est à l'érosion. Cela est dû au fait que l'érosion n'affecte pas la haute plage, qui est renforcée par le bitume. Il n'affecte que le bas de plage. On en déduit que les unités morphologiques peuvent évoluer indépendamment les unes des autres en accord avec les travaux de SABATIER (2001) qui révèlent que sur le littoral du delta du Rhône, la position du trait de côte reste fixe alors que les barres d'avant côte reculent. Les résultats obtenus et ceux des travaux antérieurs confortent la menace que fait peser l'érosion sur les infrastructures qui jouxtent le rivage. En revanche, la plage de Jacqueville s'engraisse $\left(+2300 \mathrm{~m}^{3}\right)$ et prograde. On l'estime à environ $1 \mathrm{~m} \mathrm{an}^{-1}$. Cette plage serait alimentée par les apports des secteurs voisins en érosion. L'engraissement de cette plage devrait être utile dans la perspective d'un rechargement de la baie de Port-Bouët. La distribution des faciès sur les deux sites révèle l'existence de deux stocks sédimentaires. Un stock constitué principalement de matériels grossiers, émoussés et luisants à Jacqueville, ce qui met en évidence l'influence d'un long transport par l'eau et de la dynamique fluviomarine. A Port-Bouët, l'étude montre un stock constitué en général de matériels très grossiers, subanguleux à anguleux. La présence de ces grains non (ou peu) usés reflète une source d'apport proche. A PortBouët, le materiel sableux indique des valeurs de tailles plus élevées que celles signalées par YACE (1987) et ABE (2005). Cela peut être attribué aux tempêtes exceptionnelles du mois d'août 2007 au cours desquelles s'est produit un dépôt temporaire de matériel sableux sur la plage. Ces tempêtes devraient favoriser le dépôt de sédiments très grossiers sur la plage pendant le jet de rive du fait de la très forte énergie des vagues. Par contre, lors du déplacement vers le large, les vitesses de courant étant faibles, la fraction des sédiments grossiers n’a pu être entraînée vers le large. L'action des vagues se traduit ainsi par un tri sédimentaire, le sédiment fin est vanné vers le large alors que le sédiment grossier s’accumule sur la côte (ZENKOVICH, 1946 ; MURRAY, 
1967). Les différents stocks identifiés entre Abidjan et Jacqueville sont en relation avec les facteurs naturels et les bancs rocheux signalés en face de Jacqueville auxquels il faut ajouter les travaux d'aménagement réalisés au niveau de Port-Bouët, notamment la digue d'arrêt des sables. Si aucun aménagement spécifique n’est réalisé, la baie de PortBouët va continuer à subir le phénomène d'érosion qui menace les aménagements touristiques et les infrastructures socioéconomiques et, la plage de Jacqueville s'ensablera à un rythme rapide. Ces informations s'avèrent utiles dans la gestion de cet espace littoral.

\section{Références bibliographiques}

ABE J., N’GUESSAN L. (1995). Vulnérabilité du littoral ivoirien aux changements climatiques et à l'élévation du niveau de la mer. Atelier régional sur la gestion intégré des zones littorales CERESCOR, $11 \mathrm{p}$.

ABE J., KABA N. (1997). Côte d'Ivoire, profil environnemental de la zone côtière. Ed. CEDA, 75 p.

ABE J. (2005). Contribution à la connaissance de la morphologie et de la dynamique sédimentaire du littoral ivoirien (cas du littoral d'Abidjan). Essais de modélisation en vue d'une gestion rationnelle. Thèse de doctorat d'état, Université Abidjan, 309 p.

AKA K. (1991). La sédimentation quaternaire sur la marge de Côte d'Ivoire : Essai de modélisation. Thèse de doctorat d’état, Université Abidjan, n¹46, 233 p.

AKOBE A.C. (2010). Caractérisation des bancs de grès et beach-rocks de la Côte d'Ivoire et du Ghana (Golfe de Guinée) : environnement de dépôt, maturité et pétrophysique. Thèse, Université de Cocody, Abidjan ; 166 p.

BRABANT M. (2003). Maîtriser la topographie, des observations au plan. Eryolles, $539 \mathrm{p}$.

CAILlEUX A. (1943). Distinction des sables marins et fluviatiles. Bull. Soc. Géol. France, $5^{\text {ème }}$ série, t. XIII, pp 125-138.

DELOR C., DIABY I., SIMEON Y., YAO B., TASTET J.P., VIDAL M., CHIRON J.C., DOUMANGER A. (1992). Notice explicative de la carte géologique à 1/200000, Feuille de Grand-Bassam. Mémoire n 4, 24 p.

DESMAZES F. (2000). Etude relative à la caractérisation et la répartition des barres sableuses pré-littorale. Rapport SHOM, 36 p.

DESMAZES F. (2001). Evolution morpho-sédimentaire du prisme littoral aquitain. Mémoire de DEA, Université Bordeaux 1, DGO.

DESMAZES F. (2005). Caractérisation des barres sableuses d'une plage de la côte Aquitaine. Exemple de la plage du Truc Vert. Thèse, Université Bordeaux 1, 299 p.

FOLK R.L., WARD W.C. (1957). Brazos River bar: a study in the significance of grain size parameters. J. Sedim. Petrol., Tulsa (Okl.), Vol. 27 (1), pp 3-26.

FRIEDMAN G.M. (1967). Dynamic processes and statistical parameters compared for size frequency distribution of beach and river sands. J. Sed., Vol. 37, pp 327-354. 
HAUHOUOT C. (2000). Analyse et cartographie de la dynamique du littoral et des risques "naturels" côtiers en Côte d'Ivoire. Thèse, Université Nantes, 289 p.

HAUHOUOT C. (2008). Grand-Lahou, une côte de Côte d'Ivoire en danger. Ann. Université de Lomé, Sér. Lett, Tome XXVIII-1, pp 215-222.

HINSCHBERGER F. (1977). Aspects et problèmes d'aménagement du littoral en Côte d'Ivoire. Cahier nantais, 1977, $\mathrm{n}^{\circ}$ 13, pp 51-63.

IBE A.C., QUELENNEC R.B. (1989). Méthodologie d'inventaire et de contrôle de l'érosion dans la région de l'Afrique de l'ouest et du centre. Rapport et étude sur les mers régionales, $\mathrm{n}^{\circ} 107$, in Mers régionales, $107 \mathrm{p}$.

KOFFI K.P., ABE J., AFFIAN K. (1987). Evolution actuelle du littoral ivoirien. Résultats préliminaires du suivi 1985-1987. Centr. Rech. Océanogr., Abidjan, 7 p.

LINDHOLM R.C. (1987). A pratical approach to sedimentology. London: Allen \& Unwin, 276 p. http://dx.doi.org/10.1007/978-94-011-7683-5

LIPPMANN T.C., HOLMAN R.A. (1990). The spatial and temporal variability of sand bar morphology. Journ. of Geophysic. Res. C7, pp 11575-11590. http://dx.doi.org/10.1029/JC095iC07p11575

MARTIN L. (1973). La sédimentation actuelle sur le plateau continental de Côte d'Ivoire. Cah. ORSTOM, sér., Géol., Vol. V, n² 2, pp 155- 167.

MC LAREN P. (1981). An interpretation of trends in grains size measures. J. Sedim. Petrol., Tulsa (Okl.), Vol. 51(2), pp 611-624.

MONDE S. (1997). Nouvelles approches de la cartographie du plateau continental de Côte d'Ivoire : aspects morphologiques et sédimentologiques. Thèse, Université de Cocody, Côte d'Ivoire, 175 p.

MURRAY M. (1967). Control of grain dispersion by particle size and wave state. Journal of Geology, Vol. 75, pp 612-634. http://dx.doi.org/10.1086/627285

N'DOUFOU G.H.C. (2005). Evolution du trait de côte en Côte d'Ivoire (Cas de GrandLahou). Mémoire DEA Université de Cocody, 87 p.

PELNARD C.R. (1973). Erosion à l'est du canal de Vridi. Rapport BCEOM - Port d'Abidjan, $35 \mathrm{p}$.

ROBIN M. (2002). Télédétection et modélisation du trait de côte et de sa cinématique. In Le littoral : regards, pratiques et savoirs. Éditions Rue d’Ulm/Presses de l'École Normale Supérieure, Paris, 230 p.

ROCHELEAU M. (1997). Sédimentologie des paléoplages de la plaine d'Old Crow, territoire du Yukon, Canada. Thèse, Université d'Ottawa, 168 p.

SAAIDI E. (1991). Traité de sédimentologie. Ed. Ellipses, 393 p.

SABATIER F. (2001). Fonctionnement et dynamiques morpho-sédimentaires du littoral du delta du Rhône. Thèse, Université Aix-Marseille III, 268 p.

STIVE M.J.F., DEVRIEND H.J. (1995). Modelling shoreface profile evolution. Marine Geology, 126: pp 235-248. http://dx.doi.org/10.1016/0025-3227(95)00080-I 
TASTET J.P. (1972). Quelques considérations sur la classification des côtes. La morphologie côtière. Ann. Université Abidjan (c), VII, 2, pp 135-162.

TASTET J.P. (1979). Environnements sédimentaires et structuraux quaternaire du littoral du golfe de Guinée (Côte d'Ivoire, Togo, Bénin). Thèse, Université Bordeaux 1, $\mathrm{n}^{\circ} 621,175 \mathrm{p}$.

TASTET J.P. (1987). Effets de l'ouverture d'un canal d'accès portuaire sur l'évolution naturelle du littoral d'Abidjan (Afrique de l'Ouest). Bull. Inst. Geol. Bassin d'Aquitaine, Bordeaux, $\mathrm{n}^{\circ}$ 41, pp 177-190.

TASTET J.P., CAILLON L., SIMON B. (1985). La dynamique sédimentaire littorale devant Abidjan: impact des aménagements. Contribution à la compréhension des phénomènes d'érosion et de sédimentation. Rapp. Min., Marine, Abidjan, 39 p.

VARLET F. (1958). Le régime de l'atlantique près d'Abidjan, Côte d'Ivoire. Essai d’océanographie littorale, extrait des études éburnéennes, t. 7, 222 p.

VATAN A. (1967). Manuel de sédimentologie. Éditions Technip. Paris, 397 p.

YACE P. (1987). Evolution du trait de côte et dynamique sédimentaire du littoral ivoirien entre Grand Bassam et Jacqueville. Thèse, Université Abidjan, n 105, 114 p.

ZEIGLER J.M., TUTTLE S.D. (1961). Beach changes based on daily measurements of four cape cod beaches. J. Geol., Chicago, Vol. 69(5), pp 583-599. http://dx.doi.org/10.1086/626772

ZENKOVICH V. P. (1946). On the study of shore dynamics. Akademiya nauk. USSR Institut. Okéandogi, Trudy, Vol. 1, pp 99-112. 